\title{
Research on High Pressure Oxygen Supply Box Type Intelligent Fermenter
}

\author{
Lijian Zhang ${ }^{1, a}$, Na lin ${ }^{2, b}$, Jie Li ${ }^{3, b}$, Xianmin Zhang ${ }^{4, b}$, \\ Yuexin Shang ${ }^{4, b}$, Lu Xue $e^{4, b}$ \\ ${ }^{I}$ Binzhou Polytechnic, College of Information Engineering, Shandong, China \\ ${ }^{2}$ Binzhou Polytechnic, Office of Academic Research, Shandong, China \\ ${ }^{3}$ Binzhou Bohai science and technology vocational secondary school, Shandong, \\ China \\ ${ }^{4}$ Binzhou Lianhua College, Shandong, China \\ axianruibrother@163.com,b41350992@qq.com
}

\begin{abstract}
the current situation of soil resources urgently needs to supplement soil organic matter through human measures to ensure the level of agricultural cultivation and the quality of crop products. However, many reasons, such as the lack of advanced production technology and cheap fermentation bacteria, lead to infectious diseases and insect pests, fermentation burning seedlings, soil hypoxia and bad environmental pollution in breeding areas and areas, which seriously affect the normal working and living environment of residents in surrounding areas. The project adopts high-temperature aerobic fermentation process. After fermentation, it is piled up and aged, and then bagged after being treated by powder organic fertilizer production line. It has good social, economic and ecological benefits for increasing soil fertility, improving the quality of agricultural products and increasing agricultural income.
\end{abstract}

Keywords: PLC, touch screen, device, control system

\section{INTRODUCTION}

In the process of intensive livestock and poultry breeding, if the feces are not effectively treated, a large number of harmful gases such as NH3, stink, $\mathrm{CH} 4$ will be produced after the fermentation of livestock and poultry feces. These gases will not only affect the growth of animals, but also seriously affect human health and the surrounding environment.

Over the years, the application of chemical fertilizers has played a key role in increasing agricultural production and income. However, due to the long-term application of chemical fertilizer, the supply of organic fertilizer is insufficient, 
resulting in the destruction of farmland ecological environment, soil physical and chemical properties and soil microflora to varying degrees, affecting the quality of agricultural products to a certain extent.

\section{WORKING PRINCIPLE}

The high pressure oxygen supply box type intelligent fermentor is used to mix organic wastes and auxiliary materials (sawdust, rice husk, straw, corn straw, corn straw, corn cob, peanut shell, edible fungus rod, etc.) in a certain proportion, and add microbial agents to enter the processing equipment with fermentation conditions. When the temperature and oxygen content of the material are reached, the waste material is mixed in a certain proportion, The waste organic matter of waste manure is decomposed continuously through high pressure air supply system to the material. Under the function of aerobic fermentation, the waste organic matter of manure is decomposed continuously to generate high temperature, which promotes the evaporation of water in the material, and at the same time, it kills pathogens, parasites and weeds seeds at high temperature, and achieves harmless, decrement and stabilization treatment, Meanwhile, the organic fertilizer with microbial activity was produced.

\section{CONTROL SYSTEM}

This system needs to achieve remote monitoring and intelligent control, so it needs to transfer data through the cloud platform, and it needs to design and develop the mobile client to meet the function of remote monitoring and control anytime and anywhere. At the same time, because of the need to collect temperature information and monitor the area, the system needs to design the hardware.
PLC local centralized control, analog control, sequence control and data acquisition functions. The control cabinet is installed on the equipment operation site. The signals of local instruments, valves, compressors and other equipment are sent to the control cabinet through wired way. The touch screen and buttons are installed on the control cabinet to realize the local control function.

Ethernet communication function, connect the communication line to the monitoring room, the computer runs the monitoring software, the operator can operate in the upper computer, realize the remote monitoring and control of the equipment. HMI display can be easily completed by configuration editing software. The status of switching value and the data of digital value in PLC can be dynamically displayed by text or graphics, and the user's switching value command and preset data of digital value can be input to PLC. The PLC and HMI of the automatic control system interact with each other automatically. In the system, man-machine interface information includes operation monitoring, signal status, output forcing, time information, user login and parameter setting.

Integrate today's cloud technology platform, develop integrated network monitoring system, communicate through Ethernet, WiFi, GPRS and other ways. Customers can control the field equipment through the cloud platform remote network to ensure its normal operation, so that customers can monitor the field equipment or instruments through the browser and mobile app anywhere, and obtain the real-time data, historical data, alarm information, parameter setting and other functions of the remote fermentation equipment. 


\subsection{Equipment description}

2.1.1 controlled load (PLC output)

1. 10 aeration valves $(1 \#-10$ \# fermentation tank each, air circuit solenoid valve $\mathrm{AC} 220 \mathrm{~V}$, power on and power off)

2. Two fans (interlock with ammonia concentration, operate when it is higher than $10 \mathrm{ppm}$, stop when it is lower than $10 \mathrm{ppm})$. Start value adjustable)

3 . There are 10 ways of operation / stop indication $(1-10 \times$ fermentation tank, 1 channel each.)

\subsubsection{Button switch sensor (PLC input} signal)

1. Temperature acquisition module, 1 set. 485id: 2 (the module has 10 channels in total, 3 sensor probes in each channel, and 30 DS18B20 probes in total. Shanghai soubo (SM1600)

2. Air pressure sensor, 1 set. 485id: 3 (dc12-36v, 0-1mpa, RS485 output, BSI) to collect gas pressure.

3. Gas flow sensor, 1 set. $485 \mathrm{id}$ : 4 (dv24 V, RS485 output, Nanjing Ruiji). Collect instantaneous flow and cumulative flow.

4. Ammonia gas sensor, 4 sets. 485ID : 5 、 6、7、8

5. Air compressor, 1 set, 485id: 9 (collect air supply pressure, exhaust temperature, operation time, host A-phase current, operation status)

\subsubsection{PLC and touch screen network port} communication

1 screen, (on-site display, 10 inch Kunlun Tongtai human-machine interface, display 1 and 2 fermentor information)

2 screens, (on-site display, 10 inch Kunlun Tongtai human-machine interface, displaying 3 and 4 fermentor information)

3 screen, (on-site display, 10 inch
Kunlun Tongtai human-machine interface, display 5 and 6 fermentor information)

4 screens, (on-site display, 10 inch Kunlun Tongtai state human-machine interface, displaying 7 and 8 fermentation tank information)

5 screen, (on-site display, 10 inch Kunlun Tongtai man-machine interface, display 9 and 10 fermentor information)

6 screen, (central control room display, 15 inch Kunlun Tongtai human-machine interface, display 1-10 fermentation tank information)

\subsection{Control requirements}

(1) Manual: each fermentation tank (10 fermentation tanks in total) can manually open the aeration valve (touch screen control).

(2) The automatic process is as follows:

A. Start: press the "start fermentation" button $\rightarrow$ the fermentation time is cleared and the timing starts again, and the program starts to run. At this time, it starts to collect the upper, middle and lower temperature values in the fermentation tank. And the corresponding green light is on.

B. Program operation: collect the values of three temperature sensors, compare the average temperature with the set temperature (temperature adjustable), when it is greater than the set temperature, automatically open the aeration valve, and record the aeration time, when it reaches the set aeration time (time adjustable), automatically close the aeration valve.

C. End of the program: when the fermentation time (settable) reaches the set number of days (a fermentation cycle of 7-13 days, adjusted according to the temperature in summer and winter), the collection of temperature value will be stopped automatically, and the green light will be off. 
(3) Parameter acquisition:

A. Gas pressure, curve

B. Gas instantaneous flow, curve

C. Cumulative gas flow,

D. Temperature, the on-site display screen shows the upper, middle and lower temperature curves, and the central control room display screen shows the average temperature curve of each fermentation tank.

E. Ammonia, curve

\section{CONCLUSION}

The technology adopted in the project is mature, reliable and economically reasonable. The implementation of the project has obvious economic and social benefits. And the project investment is small, the effect is fast, and the national policy support, has rich economic benefits and extensive social benefits, is conducive to improve the economic strength of the enterprise and enhance the corporate image of social responsibility.

\section{REFERENCES}

[1] Discussion on the treatment and comprehensive utilization of chicken manure in chicken farms [J]. Wang Xun, Tang Yanfei, Liu Juxiang, Wei Zonghai, Su Lishui, Su Jialian. Guangxi animal husbandry and veterinary. 2020 (01)

[2] Current situation and development trend of livestock manure fertilizer utilization in China [J]. Zhang Yuhai, Tu Yue, Chen Su, Zhang Hualong, Hu Haidong, Gao Jianying. Northeast Agricultural Sciences. 2019 (05)

[3] Analysis on aerobic composting technology of livestock manure [J]. Zhang Yifan. Livestock industry. 2019 (12)

[4] Effects of different $\mathrm{C} / \mathrm{N}$ ratios on biogas production of chicken manure by anaerobic fermentation under medium and high temperature $[\mathrm{J}]$. Li Shulan, Liu Ping,
Mei Zili. Biogas of China. 2018 (05)

[5] Pollution status, treatment and resource utilization of livestock manure in China $[\mathrm{J}]$. Wu Haowei, sun Xiaoqi, Liang Bowen, Chen Jiabin, Zhou Xuefei. Journal of agricultural environmental science. 2020 (06)

[6] J. He, X. Liu,et al. High Annealing Stability of InAlZnO Nanofiber Field-Effect Transistors with Improved Morphology by Al Doping. Journal of Physical Chemistry Letters, 12.4(2021)1339-1345.

[7] H. Zu, Y. Chang, H. Li, et. al., Modulating the Transport Properties of Metal Oxide Nanofibers Transistors by Controlling the Grain Size. IEEE Electron Device Letters. 42.6(2021)855-858.

[8] D. Liu, B. Yu, M. Liao, et. al.,Self-Powered and Broadband Lead-Free Inorganic Perovskite Photodetector with High Stability, ACS Applied Materials \& Interfaces, 12.27(2020)30530-30537. 